\title{
The Milky Way and Andromeda galaxies in a constrained hydrodynamical simulation: morphological evolution
}

\author{
Cecilia Scannapieco ${ }^{1}$, Peter Creasey ${ }^{1}$, Sebastián E. Nuza ${ }^{1}$, Gustavo Yepes ${ }^{2}$, \\ Stefan Gottlöber ${ }^{1}$, and Matthias Steinmetz ${ }^{1}$ \\ ${ }^{1}$ Leibniz-Institut für Astrophysik Potsdam (AIP), An der Sternwarte 16, 14482 Potsdam, Germany \\ e-mail: cscannapieco@aip.de \\ 2 Grupo de Astrofísica, Universidad Autónoma de Madrid, 28049 Madrid, Spain
}

Received 10 December 2014 / Accepted 27 February 2015

\begin{abstract}
Aims. We study the two main constituent galaxies of a constrained simulation of the Local Group as candidates for the Milky Way (MW) and Andromeda (M31). We focus on the formation of the stellar discs and its relation to the formation of the group as a rich system with two massive galaxies, and investigate the effects of mergers and accretion as drivers of morphological transformations. We also assess the effects of varying the assumed feedback model on our results by running two different simulations, a first one where only supernova feedback is included and a second where we also model radiation pressure from stars.

Methods. We use a state-of-the-art hydrodynamical code which includes star formation, feedback, and chemical enrichment to carry out our study. We use our two simulations, where we include or neglect the effects of radiation pressure from stars, to investigate the impact of this process on the morphologies and star formation rates of the simulated galaxies.

Results. We find that the simulated M31 and MW have different formation histories, even though both inhabit, at $z=0$, the same environment. These differences directly translate into and explain variations in their star formation rates, in situ fractions, and final morphologies. The simulated M31 candidate has an active merger history, as a result of which its stellar disc is unable to survive unaffected until the present time. In contrast, the MW candidate has a smoother history with no major mergers at late times, and forms a disc that grows steadily; at $z=0$ the simulated MW has an extended, rotationally-supported disc that is dominant over the bulge. Our two feedback implementations predict similar evolutions of the galaxies and their discs, although some variations are detected, the most important of which is the formation time of the discs: in the model with weaker (stronger) feedback the discs form earlier (later). In summary, by comparing the formation histories of the two simulated galaxies, we conclude that the particular merger and accretion history of a galaxy rather than its environment at the LG-scales is the main driver of the formation and subsequent growth or destruction of galaxy discs.
\end{abstract}

Key words. galaxies: formation - galaxies: evolution - galaxies: structure - cosmology: theory - methods: numerical

\section{Introduction}

The formation of late-type spiral galaxies in the context of the $\Lambda$-cold dark matter $(\Lambda \mathrm{CDM})$ cosmological framework is a longstanding problem in astrophysics. In hierarchical models such as $\Lambda \mathrm{CDM}$, the presence or absence of a strong, rotationally supported stellar component is influenced by processes acting at many scales as galaxies evolve; baryons condense in the centres of dark matter haloes whilst retaining a considerable fraction of their angular momentum set at large scales (Fall \& Efstathiou 1980) and a combination of viscous and dissipative processes in the gaseous component allows the formation of a disc from which a fraction is converted into a stellar component. Thicker stellar components result from dynamical processes such as disc instabilities (Sellwood \& Binney 2002) and the close encounters and mergers (e.g. Quinn et al. 1993) that are ubiquitous in hierarchical growth models such as $\Lambda \mathrm{CDM}$, though some simulations (e.g. Bird et al. 2013) alternatively suggest that the older stellar discs may have been born thick.

Numerical simulations are the best tool with which to study galaxy formation within $\Lambda \mathrm{CDM}$, and to investigate the relationship between the formation of stellar discs and the hierarchical build-up of the haloes containing them (Scannapieco et al. 2009; Sales et al. 2012). During recent years, important progress has been made in the field, and simulations currently appear to be reaching the level of sophistication required to study the details of the assembly of galaxies and their stellar discs. In fact, while a first generation of models inevitably suffered from the angular momentum catastrophe (e.g. Navarro \& Benz 1991; Steinmetz \& Navarro 1999), more recent codes showed that various types of feedback provide galaxies with self-regulated star formation activity, in turn allowing the formation of young, rotationallysupported stellar discs (Scannapieco et al. 2012). Feedback processes produce a decoupling between the formation of a gas disc after its condensation within a halo and the onset of star formation activity; in this way, stellar discs can form later, in more quiescent periods rather than during the early, violent times characteristic of the formation of the haloes. When discs form later, they also have a greater chance to survive and can grow to form extended, highly-rotating systems by $z=0$ (Scannapieco et al. 2009). At the heart of the problem of disc formation/survival is therefore the problem of understanding how star formation proceeds in galaxies, and what the possible regulating mechanisms and their relative efficiencies are. 
For Milky Way-mass galaxies, the most efficient feedback mechanism is believed to be that produced by stars, via the injection of energy into the interstellar medium during different phases of stellar evolution. In particular, various models for feedback from supernovae ( $\mathrm{SNe}$ ) have been included in simulations in the last decade, and demonstrated that SN feedback is indeed capable of regulating star formation and of producing stellar discs with high angular momenta (e.g. Okamoto et al. 2005; Governato et al. 2007; Scannapieco et al. 2008, 2009; Piontek $\&$ Steinmetz 2011). However, stellar discs in these models are usually subdominant over the massive bulges that form early. The more recent inclusion of additional feedback in the form of radiation pressure produced by massive stars showed that this process provides significant kinetic energy to the gas, particularly at early times, helping to produce even younger, more massive galaxy discs similar to those observed (Aumer et al. 2013; Stinson et al. 2013; Hopkins et al. 2014; Agertz \& Kravtsov 2014).

Despite the recent progress, the Aquila code comparison project (Scannapieco et al. 2012) showed that, even when the formation history of a halo is fully specified, different models predict the formation of a galaxy with different gas fractions, morphologies, stellar masses and angular momentum content. Aquila clearly demonstrated that the primary uncertainty in these types of simulations is related to the processes of the unresolved baryonic physics that result in star formation and feedback and their respective modelling, and generated consensus on the need to produce similar results using different codes, despite their individual successes. Efforts to overcome these problems are indeed ongoing, both in terms of direct comparison between codes (Kim et al. 2014) and of the development of more physicallymotivated routines to treat star formation and feedback (e.g. Christensen et al. 2012; Agertz et al. 2013; Aumer et al. 2013; Creasey et al. 2013; Stinson et al. 2013; Vogelsberger et al. 2013; Agertz \& Kravtsov 2014; Ceverino et al. 2014; Hopkins et al. 2014; Murante et al. 2015; Trujillo-Gomez et al. 2015).

An important motivation to study the formation of late-type spiral galaxies is the membership of our own galaxy, the Milky Way (MW), within that family. The MW provides an ideal place to constrain galaxy formation theories, as we have detailed observational data on a large number of stars residing in its different stellar components. A salient characteristic of the MW is that it lives in a group environment, known as the Local Group (LG), with another large spiral, Andromeda (M 31), lying at less than a Mpc distance from it. A number of observational studies showed that the immediate environment of galaxies indeed plays a role in the determination of their properties at the scales of galaxy clusters and rich groups (e.g. Dressler 1980; Hermit et al. 1996; Guzzo et al. 1997; Blanton et al. 2003; Girardi et al. 2003), although for systems like the LG this is more controversial (e.g. Bahé et al. 2013; Ziparo et al. 2013). On the other hand, simulation studies have almost exclusively used initial conditions (ICs) that follow the formation of a galaxy which is isolated from massive neighbours at the Mpc scales (see, however, Few et al. 2012). A study on possible effects of environment on the formation of the MW and M31 is therefore required in order to understand if these are representative of galaxies of a similar mass and to better interpret observational results.

In this work, we use constrained ICs of the Local Universe, where a LG-like pair of galaxies form. We study the formation and evolution of these two LG-galaxies, candidates for the MW and M 31, using a state-of-the-art hydrodynamical code for cosmological simulations. We use these simulations to investigate how the LG galaxies could have formed, and to look for possible imprints of the formation of the LG on their final properties. In particular, we focus on the formation of the main stellar components of the galaxies, and investigate possible correlations between the formation of stellar discs and large-scale effects such as merger events and misalignment between the gaseous and stellar discs. The main questions we want to address here are whether our simulation predicts the formation of two large spiral galaxies similar to the MW and M 31 and what are the mechanisms determining/affecting their formation. We will show that only the simulated MW has a stable stellar disc that survives to $z=0$; while in M31 various mergers continuously affect the disc, and it produces a galaxy with a much lower level of rotational support. We find similar results using two different versions of the code that we use to test whether results are affected by the particular treatment of feedback processes. It is worth noting that, as shown in the Aquila Project (Scannapieco et al. 2012), the final morphology of a galaxy depends sensitively on the amount of star formation during the early epochs of the formation of galaxies, and therefore on the treatment of feedback processes which regulate the star formation activity.

Other studies using our simulations are presented in a series of companion papers. Nuza et al. (2014b) analysed the properties of the neutral, warm and hot gas within and around the haloes of the MW and M 31 candidates in one of our simulations, including accretion/ejection rates of the different components and $\mathrm{HI}$ covering fractions that we contrasted to observational results. In Creasey et al. (2015), we further investigate the relation between environment and galaxy properties, by comparing the properties of our simulated MW and M 31 with those of galaxies of similar mass but formed in more isolated environments. Our findings suggest that some of the properties of galaxies, such as the star formation rates, may indeed be directly affected by the environment at the Mpc scales; while others, like the disc stellar mass fraction, are more sensitive to the merger and approach of satellite systems to the inner regions of the galaxies. (For other simulation studies analysing the relation between galaxy properties and environment in constrained simulations - albeit at much larger scales - see e.g. Nuza et al. 2010, 2014a).

The outline of our paper is as follows: in Sect. 2 we describe the initial conditions and code used for our study; Sect. 3 describes the assembly of the LG and the LG galaxies in our simulations; and in Sect. 4 we discuss the morphological transformations of the simulated galaxies during their evolution, and investigate the relation between the evolution of morphologies and the merger histories. Finally, in Sect. 5, we summarize our results.

\section{The simulations}

We use for this study a simulation designed to produce a LG-like system in the right cosmological environment, and investigate the formation of its two main constituent galaxies, candidates for the Milky Way and Andromeda. In this section we describe the numerical methods needed to carry out such a study, and summarize the numerical set-up of the simulations.

\subsection{Simulation code}

We use the Tree-PM smoothed particle hydrodynamics (SPH) code GADGET3 (Springel 2005; Springel et al. 2008), and run two different simulations where additional updates are included. The first simulation, labelled "CS" throughout this paper, uses 
the extensions of Scannapieco et al. $(2005,2006)$ for star formation, metal-dependent cooling, chemical enrichment and (thermal) feedback from Type II and Type Ia supernova (SN) explosions, a multiphase model for the gas component, and a UV background field (Haardt \& Madau 1996). The second simulation, labelled "MA", additionally includes the effects of radiation pressure from stars and kinetic feedback from SN explosions, as described in Aumer et al. (2013).

We have shown in previous work that the CS model is able to reproduce the formation of galaxy discs from cosmological initial conditions, alleviating the angular momentum problem. However, although discs have realistic sizes and angular momentum content, overly massive bulges are also formed which are usually dominant (in mass) over the discs (Scannapieco et al. 2008, 2009, 2010, 2011, 2012). The more recent MA implementation is an update to the CS model. In this case, early stellar feedback from new born stars and kinetic feedback from SN explosions is additionally considered, which leads to stronger effects particularly at high redshifts. The MA model produces galaxies with a high angular momentum content, moderate stellar masses and dominant discs, in better agreement with observations of spiral galaxies. It is therefore relevant for our work to compare the results obtained with the two models, which allows us to test the robustness of our results, particularly in relation to the effects of mergers during the evolution of galaxies.

In both models, star formation takes place in dense $\left(n_{\mathrm{H}}>\right.$ $0.03 \mathrm{~cm}^{-3}$ ) regions of converging flow, with a star formation rate per unit volume equal to

$\dot{\rho}_{\star}=c_{*} \frac{\rho}{\tau_{\text {dyn }}}$,

where $\rho$ and $\tau_{\text {dyn }}=1 / \sqrt{4 \pi G \rho}$ are, respectively, the density and dynamical time of the gas particle, and $c_{*}$ a star formation efficiency (that we set to $c_{*}=0.1$ ). Our simulations use the multiphase model described in Scannapieco et al. (2006), where the neighbour list of any particle $i$ with entropy $A_{i}$ is constructed ignoring neighbours $j$ with $A_{j}<50 A_{i}$ (provided they are not part of a shock to avoid unphysical behaviour). In this way, a multiphase medium is generated, where particles of varying densities and temperatures (i.e. cold and hot) can coexist in the same spatial region. These cold and hot phases are however not fixed, as the selection of particles to be ignored as neighbours depends on the relative properties of each pair of particles $i, j$ and is not based on any pre-fixed values for different phases. As explained in Scannapieco et al. (2006), this model allows coexistence between a dense and a diffuse phase, and also a more efficient deposition of the supernova energy.

In the CS model, each star particle can explode a maximum of two times, first as SN Type II (SNII) and later on as SN Type Ia (SNIa). During each SN explosion, the star distributes chemical elements (according to the corresponding SNII and SNIa chemical yields) and energy into neighbouring gas particles. The energy and chemical production of each exploding star is distributed in equal proportions to its hot (non-star-forming) and cold (star-forming) gas neighbours. We assume a canonical $0.7 \times 10^{51}$ erg of energy to be released per SN. The distribution of chemical elements occurs at the time of the explosion, as well as the release of energy to the hot phase. However, for the cold phase, feedback energy is accumulated in a reservoir, until it is sufficient to allow the particle to thermalize with the local hot phase. In practice, this works as follows: for each cold gas particle, we calculate the average density and entropy over its hot neighbours (those particles that were ignored in its neighbour list according to our multiphase model) and calculate the energy needed for the cold particle to reach these values, $\Delta E$. This energy is then compared to the energy stored in the reservoir $E_{\text {res }}$, and when $E_{\text {res }}>\Delta E$ the cold particle gets the reservoir energy as thermal energy. We note that the value of $\Delta E$ is different and time-dependent for each cold particle, which makes the code suitable to adapt to the cold/hot regions with different temperatures and densities, which are present at different times during the formation of a galaxy and at different spatial regions.

The MA model works similarly to the CS model; however, in this case, the $\mathrm{SN}$ energy is split into a kinetic and a thermal component. The kinetic energy transferred from the exploding star to the gas neighbours is calculated assuming that the momentum of the SN ejecta is conserved, and characterized by an outflow velocity $v_{\text {out }}: \Delta p=\Delta m \cdot v_{\text {out }}$. We use $v_{\text {out }}=3000 \mathrm{~km} \mathrm{~s}^{-1}$, with momentum given in the radial direction. As the momentum is distributed into neighbouring particles, which typically translates into a velocity kick per particle of $\Delta v_{i} \sim 20-30 \mathrm{~km} \mathrm{~s}^{-1}$. The remaining energy (i.e. the total energy released per exploding star minus the kinetic energy that has been already been transferred to the neighbouring gas particles) is stored into the thermal reservoir of the gas receiving feedback, and is treated in the same way as in the CS model. The MA model includes a treatment for radiation pressure, where the rate of momentum deposition in the gas is parametrized as

$p_{\mathrm{rp}}=\left(1+\tau_{\mathrm{IR}}\right) \frac{L(t)}{c}$,

with $L(t)$ being the UV-luminosity of the stellar population and $\tau_{\text {IR }}$ an infrared optical depth that depends on the density, metallicity, and velocity dispersion of the gas around the star. This last dependency makes the effects of radiation pressure larger in systems with higher velocity dispersions, typical of high- $z$ galaxies, allowing a more efficient regulation of star formation at early epochs and higher star formation levels at late times.

We note that, as described in Aumer et al. (2013), the MA model considers additional updates (new treatment of chemical enrichment and metal diffusion, AGB stars, and element-byelement cooling) that we do not include here, and therefore the only differences between our two simulations is the inclusion of radiation pressure and the additional kinetic feedback assumed for $\mathrm{SNe}$. In order to better compare results from our two simulations, we have also used the exact same input parameters for star formation and feedback when appropriate (which in some cases differ from those assumed in Aumer et al. 2013), and also used the same choice for the gravitational softening, which was (for the high resolution particles) $0.7 \mathrm{kpc}$, fixed in physical coordinates since $z=3$ and fixed in comoving coordinates at earlier times. In this way, any difference in the results can be directly associated with the effects due to the radiation pressure of young stars and kinetic feedback during the SN phase.

We note that some properties of the simulated galaxies, particularly the detailed final morphology, are expected to be sensitive to resolution (Scannapieco et al. 2011) and to the choice of the gravitational softening (Aumer et al. 2013). However, although higher resolution and/or lower values for the gravitational softening will allow the vertical/radial structure of discs to be better resolved, the presence or absence of a disc will not be affected by these choices (as long as a minimum resolution is considered). Our current work focuses on the evolution of discs and therefore our results are still valid, even when the spatial resolution is not high enough to resolve the radial/vertical structure of discs with a large number of resolution elements. 
Table 1. Main properties of the G1 and G2 galaxies in our two simulations at $z=0$ : radius $R_{200}$, and total, gaseous and stellar $\left(M_{200}, M_{\text {gas }}\right.$ and $M_{\text {stars }}$ ) masses within $R_{200}$.

\begin{tabular}{lcccc}
\hline \hline $\begin{array}{l}\text { Galaxy- } \\
\text { model }\end{array}$ & $\begin{array}{c}R_{200} \\
{[\mathrm{kpc}]}\end{array}$ & $\begin{array}{c}M_{200} \\
{\left[10^{10} M_{\odot}\right]}\end{array}$ & $\begin{array}{c}M_{\text {gas }} \\
{\left[10^{10} M_{\odot}\right]}\end{array}$ & $\begin{array}{c}M_{\text {stars }} \\
{\left[10^{10} M_{\odot}\right]}\end{array}$ \\
\hline G1-CS & 244.9 & 167.9 & 6.67 & 7.95 \\
G1-MA & 239.1 & 155.1 & 6.13 & 6.76 \\
G2-CS & 222.2 & 125.1 & 5.69 & 6.24 \\
G2-MA & 219.5 & 120.6 & 6.39 & 5.48 \\
\hline
\end{tabular}

\subsection{Initial conditions and simulation set-up}

The initial conditions (ICs) used for this study are constrained initial conditions of the Local Universe, which are part of the CLUES (Constrained Local UniversE Simulations ${ }^{1}$ ) project. The ICs reproduce, by construction, the known dynamical properties of our local environment at the present-day (see Gottlöber et al. 2010; and Yepes et al. 2014; for details) and use the zoomin technique, in a periodic box of $64 h^{-1} \mathrm{Mpc}$ on a side. In the centre of this cube, a high-resolution region of $2 h^{-1} \mathrm{Mpc}$ comoving radius contains dark matter and gas particles, with a mass resolution of $2.8 \times 10^{6} M_{\odot}$ and $5.6 \times 10^{5} M_{\odot}$, respectively. Outside the high-resolution region, the mass distribution is described by low-resolution collisionless particles, as usual in zoomed simulations.

The ICs are consistent with a $\Lambda$ CDM universe with WMAP-5 cosmological parameters: $\Omega_{\mathrm{M}}=0.279$ (matter density), $\Omega_{\Lambda}=0.721$ (dark energy density), $\Omega_{\text {bar }}=0.046$ (baryon density), $H_{0}=100 h \mathrm{~km} \mathrm{~s}^{-1} \mathrm{Mpc}^{-1}$ with $h=0.7$ (Hubble parameter), and $\sigma_{8}=0.8$ (normalization of the power spectrum), and correspond to a starting redshift of $z=50$.

\subsection{Milky Way and Adromeda candidates}

At $z=0$, the simulated LG has two main constituent galaxies, with total masses similar to the MW, which we take as candidates for the MW and Andromeda ${ }^{2}$. We will refer to these galaxies as G1 (candidate for M31) and G2 (candidate for MW) ${ }^{3}$ and, where appropriate, we will add an acronym CS/MA to denote the simulation run. The assignment of our galaxy candidates to the real counterparts is based on their mass, G1 being the most massive at $z=0$ (see Table 1). Additionally, with this choice, we recover a similar relative orientation between the galaxies in comparison to observations (Nuza et al. 2014b).

Table 1 shows the main $z=0$ properties of $\mathrm{G} 1$ and $\mathrm{G} 2$ in our two runs: $R_{200}$, defined as the radius where the density contrast is 200 times the critical density, and the total $\left(M_{200}\right)$, gaseous $\left(M_{\text {gas }}\right)$ and stellar $\left(M_{\text {stars }}\right)$ masses, all within $R_{200}$. The present-day masses of G1 $\left(\sim 1.7 \times 10^{12} M_{\odot}\right)$ and G2 $(\sim 1.2 \times$ $\left.10^{12} M_{\odot}\right)$ are similar to the values estimated for Andromeda $\left(\sim 1.4 \times 10^{12} M_{\odot}\right.$; Corbelli et al. 2010 ; Watkins et al. 2010$)$ and the Milky Way $\left(\sim 10^{12} M_{\odot}\right.$; Wilkinson \& Evans 1999; Smith et al. 2007; Watkins et al. 2010; Bovy et al. 2012; Kafle et al. 2012; Piffl et al. 2014) from observational studies.

\footnotetext{
1 http://www.clues-project.org/

2 As the constraints can only be imposed on large scales, the properties of the two main galaxies will not necessarily resemble those of the actual MW and M31 in detail.

3 In Nuza et al. (2014b) we instead refer to G1 and G2 as M $31^{\mathrm{c}}$ and $\mathrm{MW}^{\mathrm{c}}$, respectively.
}

\section{The assembly of the LG and the LG galaxies}

The assembly history of the LG and of the LG galaxies (G1, candidate for M31 and G2, candidate for MW) is seen in Fig. 1, where we show the dark matter, gaseous and stellar mass distributions (in an arbitrary projection) at $z=2,1,0.5$ and 0 . The different panels of Fig. 1 are centred at the centre of mass of the G1-G2 system at the corresponding cosmic epochs, and the two galaxies are surrounded by solid (G1) and dashed (G2) circles indicating their $R_{200}$ radii. At each redshift, the plots show the mass distributions in a cubic box of $2.5 \mathrm{cMpc}^{4}$ side length that corresponds to the high-resolution region. The different colours span 4 orders of magnitude in logarithmic scale, and we adopted the same colour scale at all redshifts in order to highlight time variations in the distributions 5 .

At $z=2$, the characteristic filamentary structure at Mpc scales is clearly seen; G1 and G2 are $550 \mathrm{kpc}$ apart (i.e. more than a Mpc apart in comoving coordinates), and belong to different filaments. The Local Group evolves quickly and, by $z=1$, defines a system where the mass distributions of its two main galaxies are interconnected - a feature that is particularly visible in the gas component - and have collapsed to the same filament. The two galaxies continue separating from each other until $z \sim 0.5$ when they reach a maximum separation of $\sim 900 \mathrm{kpc}$. After this time, G1 and G2 start approaching each other, to reach a final separation of about $800 \mathrm{kpc}$. The stars are located within the very centres of the dark matter haloes, resulting in much less extended distributions compared to the gaseous and dark matter counterparts. A significant number of smaller galaxies and satellites of G1 and G2 are observed, particularly at high redshift; most of them have been accreted by the G1 and G2 haloes by $z=0$.

Although G1 and G2 are, at $z=0$, both part of the simulated LG, their respective growth history are somewhat different, as shown by the evolution of their total masses and radii shown in the left-hand panels of Fig. 2. In the case of G1, the size and mass increase rapidly from $z=3$ to $z=0$, particularly after $z \sim 1$ owing to the accretion of a number of satellite galaxies ${ }^{6}$. These satellites are being accreted at approximately the same time around $z=0.9-0.7$, and contribute a significant amount of mass to G1, determining the increase and variations of $R_{200}$. Slightly after $z=0.5$, the satellite galaxies are in their last apocentre, and the evolution of G1 becomes more quiet until the present time. In contrast, G2 has a more steady evolution at all times, with the most significant changes at $z \approx 1-1.5$, which also result from the entrance of a relatively small satellite galaxy. The agreement between the evolution of G1 and G2 in our two simulations is excellent in terms of the masses and radii.

The different growth of the haloes of G1 and G2 also reflect in the assembly of the baryonic components, with a much smoother evolution of G2 compared to G1, as shown in the right-hand panels of Fig. 2. However, as the evolution of the gaseous/stellar masses are affected not only by the growth of the haloes with time, but also by the transformation of gas into stars and the return of mass from the stars to the gas owing to stellar evolution, larger differences between the two runs are

\footnotetext{
4 For short, throughout the paper we will use a prefix $\mathrm{c}$ to length units to denote the use of comoving coordinates.

5 These figures correspond to our CS run although, at these scales, the results are qualitatively the same as those obtained with the MA model.

6 We discuss the merger histories of G1 and G2 in Sect. 4.
} 
C. Scannapieco et al.: A constrained simulation of the Local Universe

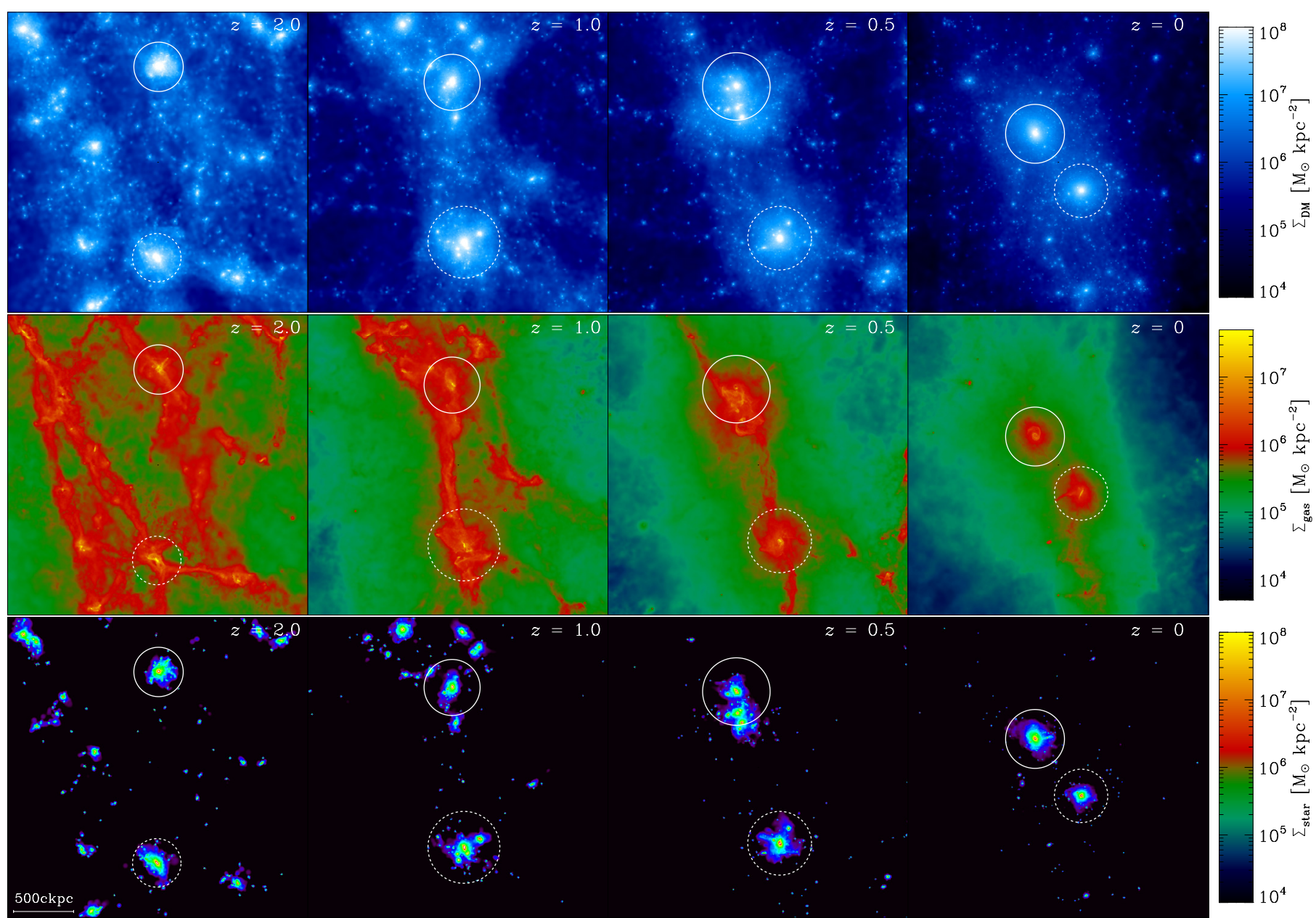

Fig. 1. Spatial distribution (in an arbitrary projection) of the dark matter (upper panels), gas (middle panels) and stars (lower panels) in our simulation, in a cube of $2.5 \mathrm{cMpc}$ on a side and for redshifts $z=2,1,0.5$ and 0 . The $R_{200}$ of G1 (solid line) and G2 (dashed line) at each redshift are indicated, and the colour scale (of each mass component) is fixed in order to highlight differences in the distributions at different times. The plots correspond to run CS, but results at these scales are very similar for both simulations.
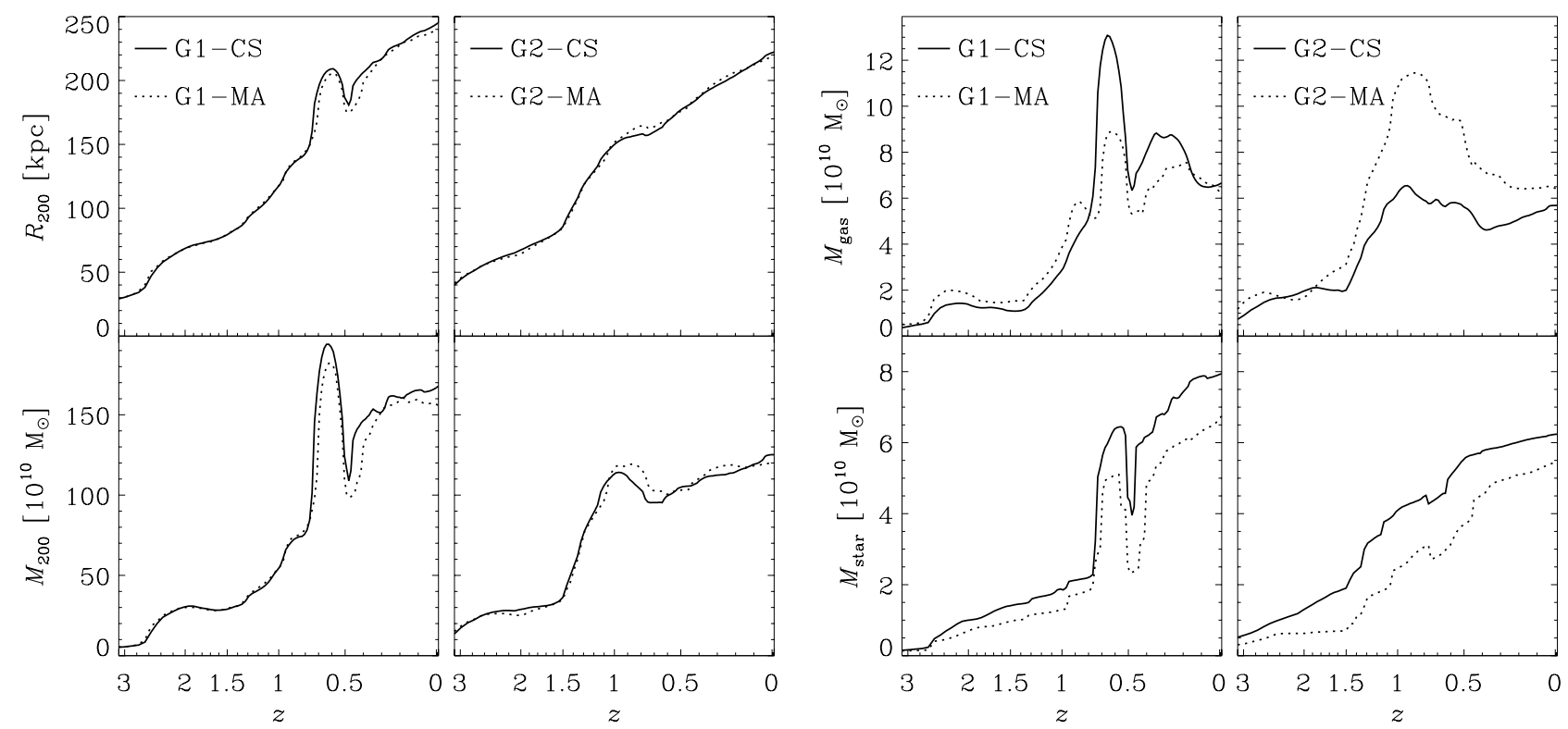

Fig. 2. Size/mass evolution of G1 (solid line) and G2 (dashed line) as a function of redshift. We show the evolution of $R_{200}$ (upper-left panels), the total mass $\left(M_{200}\right.$, lower-left panels), the gas mass ( $M_{\mathrm{gas}}$, upper-right panels) and the stellar mass $\left(M_{\mathrm{star}}\right.$, lower-right panels), all within $R_{200}$. For G1, the peak seen in all panels at $z \approx 0.65$ is owing to the emergence of two large objects within $R_{200}$ (see further discussion in Sect. 4). 

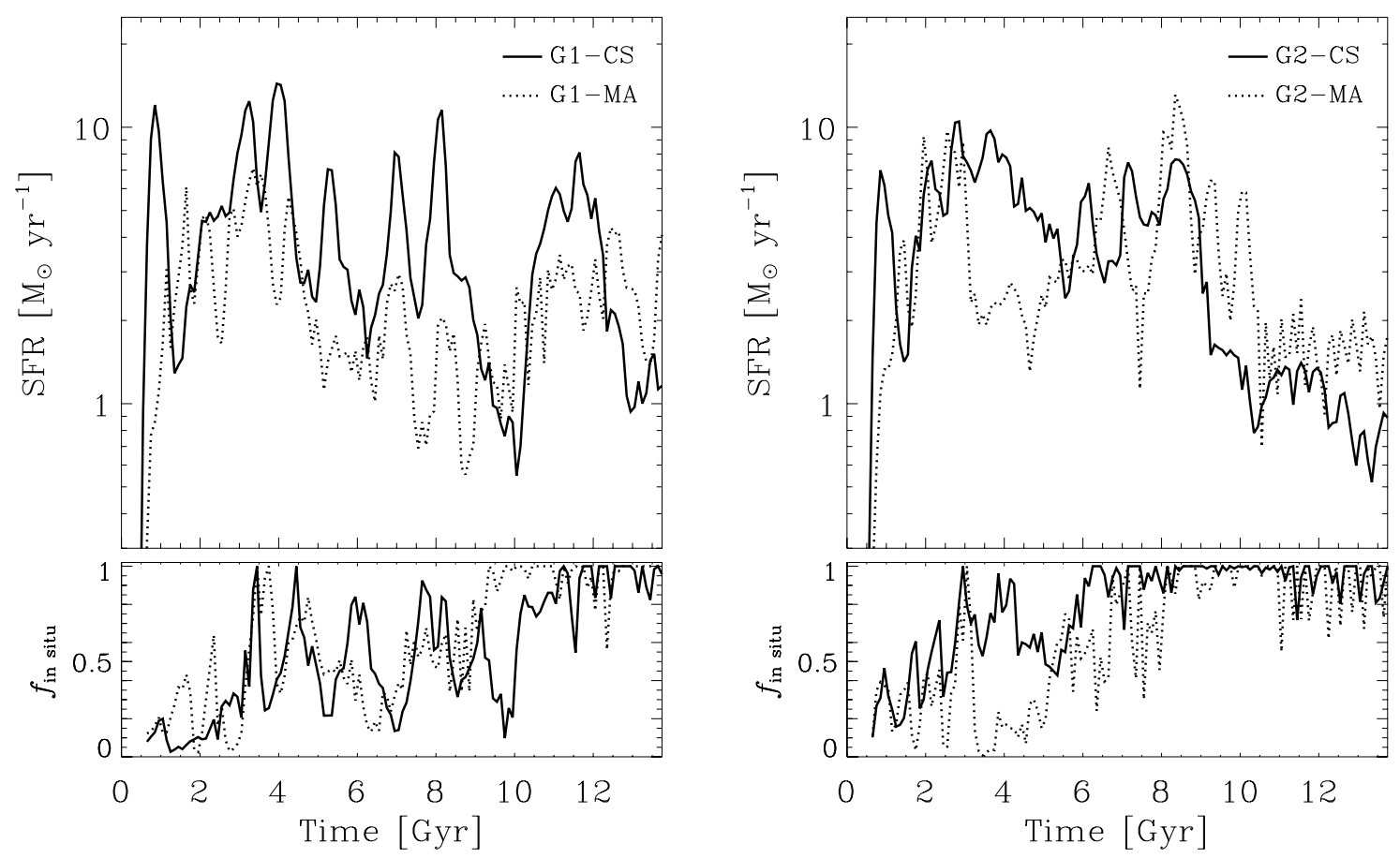

Fig. 3. Star formation rate of G1 (left-hand panel) and G2 (right-hand panel) as a function of cosmic time since the Big Bang for simulation runs CS (solid lines) and MA (dotted lines). The lower panels show the corresponding in-situ fractions, i.e. the stellar mass fraction formed in the main progenitor at each time interval.

found for the baryonic components ${ }^{7}$. As a result of the stronger feedback assumed in model MA, galaxies have in general lower stellar and higher gaseous masses in this run, compared to simulation CS. This effect is already noticeable at high redshift, because of the early feedback associated with massive, short-lived stars, which acts shortly after the onset of star formation activity in the galaxies.

The differences in stellar/gas masses between results of runs CS and MA are a consequence of the different star formation rates (SFRs), shown in the upper panels of Fig. 3. To calculate the SFRs, we considered all stars that end up in the main galaxy at $z=0$ (i.e. within $30 \mathrm{kpc}$ ), and therefore include stars formed in-situ (within the main progenitor at each time) and in satellite systems that were later accreted. The SFRs are higher at early times and gently decline at more recent epochs. Feedback effects start before in run MA compared to run CS, as radiation pressure acts right after the onset of star formation. In fact, the first star formation burst produced in run CS (in both galaxies) is absent in simulation MA. In the case of G1, we find that the SFRs in model MA are in general lower compared to model CS, with the most important differences at very early times and around 6-8 Gyr. For G2, differences are significant (additionally to the very early epochs) at 4-6 Gyr, with lower SFRs for model MA, and after 9 Gyr of evolution, with higher SFRs for model MA compared to CS.

The lower panels of Fig. 3 show the corresponding in-situ fractions, $f_{\text {in-situ }}$, which help us to understand variations between the evolution of G1 and G2 that result from the different SFRs of the progenitor galaxies and their satellites. An important difference between G1 and G2 (in both runs) shows up very clearly in these plots: G1 has much lower in-situ fractions at all times

\footnotetext{
7 However, the simulations presented here only consider mass return from SN explosions, while they do not include a treatment for the mass return of intermediate-mass stars that would result in a larger correction.
}

compared to G2. Only at low redshifts does G1 have $f_{\text {in-situ }} \sim 1$, while at earlier times there is a high contribution of stars that form in other systems than the main progenitor. In contrast, G2 has higher in-situ fractions at all times: $f_{\text {in-situ }} \sim 1$ for at least the last 6-8 Gyr. As we discuss in Sect. 4, these differences can explain the origin of the final morphologies of the simulated galaxies, as G1 has a much more active merger history which makes its stellar disc much more susceptible to partial or total destruction.

\section{Galaxy morphologies and their relation to the formation/merger history}

The figures of the previous section already revealed that G1 and G2 exhibit differences in their growth/star formation history. In this section, we investigate how these differences are translated into variations in the final properties of the galaxies, particularly in their morphologies/dynamical state.

The stellar distributions of the central, main component of $\mathrm{G} 1$ and $\mathrm{G} 2$ in our two runs and at $z=0$ are shown in Fig. 4 for face-on and edge-on views, in an area of $(40 \mathrm{kpc})^{2}$. To create these maps, we have rotated the galaxies such that the stellar angular momentum within a radius of $30 \mathrm{ckpc}$ lies in the $z$-direction. We also plot isodensity contours at fixed density values in order to highlight differences in the mass distributions of the two simulations.

Immediately apparent is that, in both runs, G1 is a more spheroidal galaxy than G2, which has a well-defined, extended, disc-like component. The presence/absence of a disc can be inferred from the projected velocity fields overplotted to the stellar densities. These show that G1 has a certain degree of rotational support that is however not very significant. Compared to G1-CS, the rotation in G1-MA is much more coherent, as shown not only by the tangential velocities in the face-on view, but also 
C. Scannapieco et al.: A constrained simulation of the Local Universe
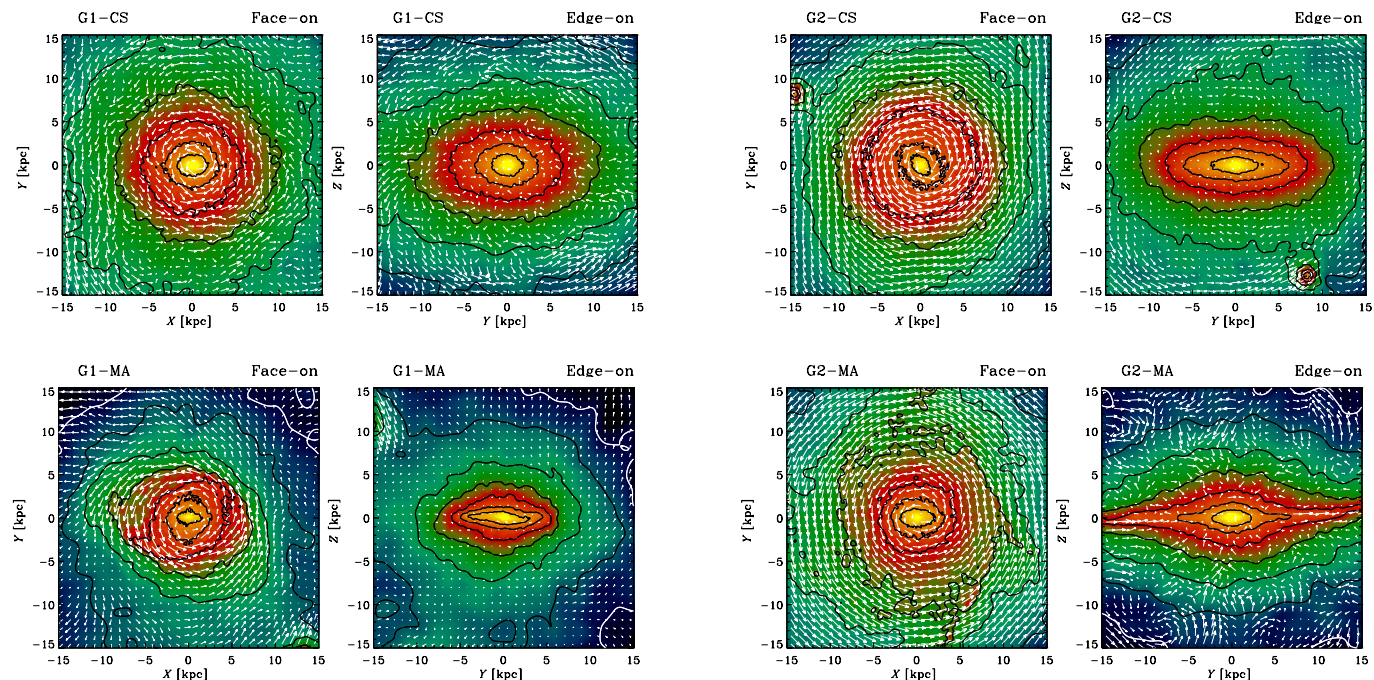

Fig. 4. Projected stellar mass density of G1 (left-hand panels) and G2 (right-hand panels) showing edge-on and face-on views for simulation runs CS (upper panels) and MA (lower panels). Also shown are the velocity field (arrows) and isodensity contours (solid lines) in the corresponding plane.
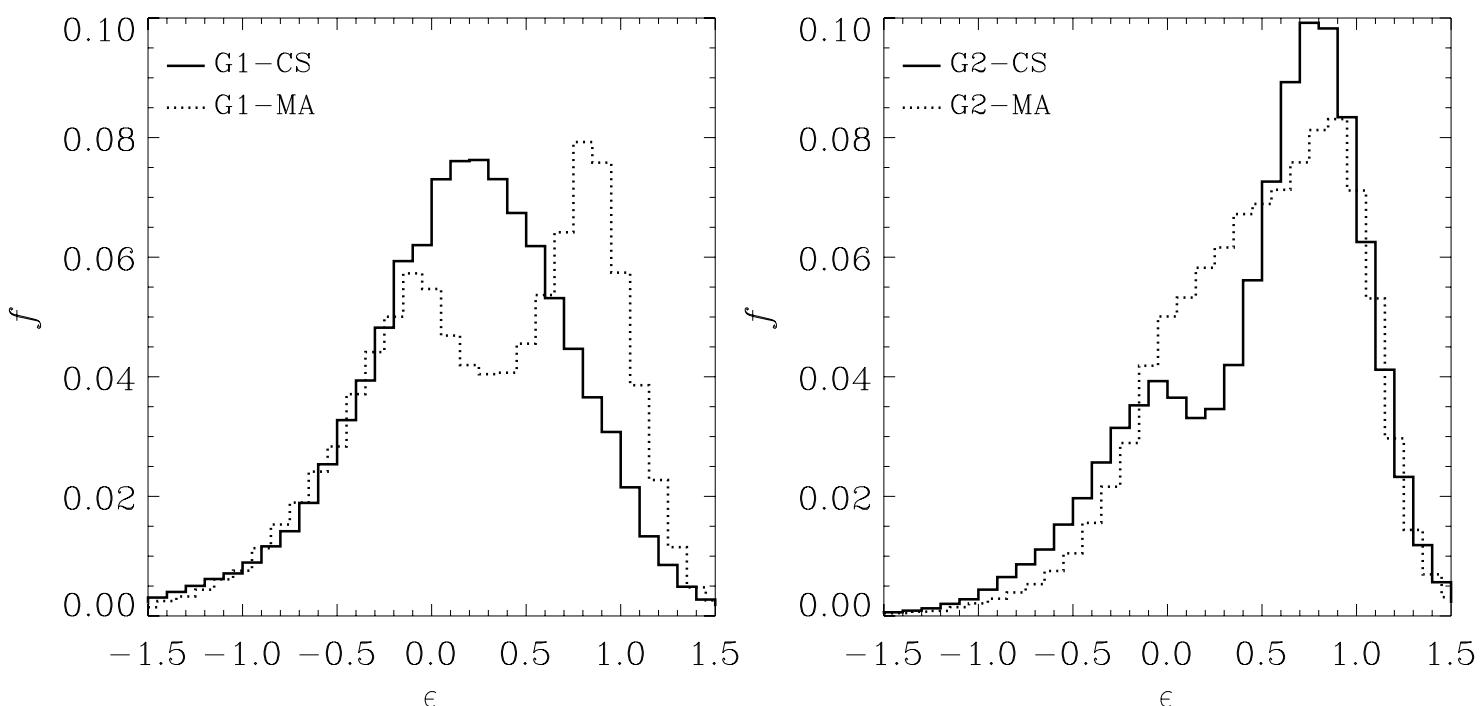

Fig. 5. Distribution of stellar circularities of G1 (left-hand panel) and G2 (right-hand panel) for simulation runs CS (solid lines) and MA (dotted lines) at $z=0$.

by the low velocities observed in the edge-on projection. A much higher degree of rotation is found for $\mathrm{G} 2$ in both simulations, where a well-defined, extended disc component is present. As shown later (Figs. 5 and 7) neither G2-CS nor G2-MA has a dominant, massive bulge in the centre, and neither shows evidence of a bar. We note, however, that because of to its younger age (Fig. 7, see below), the disc of G2-MA is thinner compared to that of G2-CS. We also note that, both for G1 and G2, we find that the stellar haloes are more extended in run CS compared to run MA, which is a direct consequence of the stronger feedback assumed in the latter which prevents the formation of the old stars that make up this component.

In order to better quantify the dynamical state of G1 and G2 and compare results from our two simulations, we show in Fig. 5 the corresponding distributions of stellar circularities. The circularity of a star $i$ is defined as in Scannapieco et al. (2009),

$\epsilon_{i} \equiv \frac{j_{z}^{i}}{j_{\text {circ }}\left(r_{i}\right)}$ where $j_{z}^{i}$ is the angular momentum of the $i$ star particle perpendicular to the disc plane (i.e. in the $z$-direction), and $j_{\text {circ }}$ the angular momentum expected for a circular orbit at the star's radius. Circularities of the order of 1 are expected for rotationallysupported particles while spheroidal components form more or less symmetric distributions around $\epsilon=0$ (non-rotating bulges), $0 \lesssim \epsilon \lessgtr 0.5$ (rotating bulges) or $-0.5 \lessgtr \epsilon \lessgtr 0$ (counter-rotating bulges).

As inferred from Fig. 4, Fig. 5 confirms that G1 has an important spheroidal-like component. In G1-CS, the spheroid has an overall rotation (the peak of the distribution is at positive $\epsilon$ ), while in G1-MA the spheroid does not rotate. Furthermore, G1-MA has a disc-like component in rotational support. As we show later, the presence/absence of the disc in G1-CS relates to the fact that this galaxy has experienced, before $z=0$, a number of mergers which destroyed a pre-existing disc, while these events have not yet happened in G1-MA at $z=0$. In the case of $\mathrm{G} 2$, the presence of a well-defined, rotationally-supported disc 

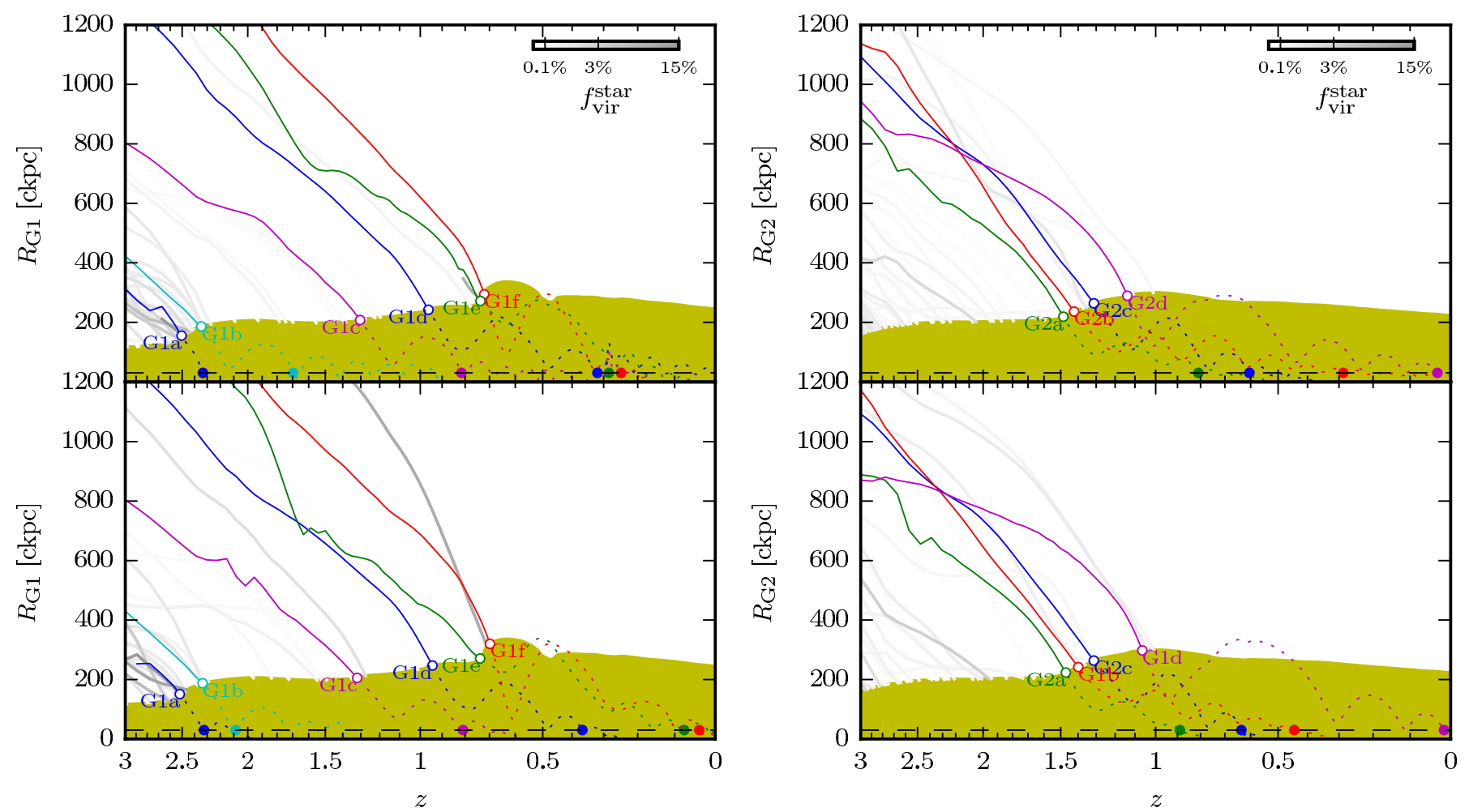

Fig. 6. Merger history of G1 (left-hand panels) and G2 (right-hand panels) for simulations CS (upper panels) and MA (lower panels), alongside the evolution of its statistics. The plots show the comoving distance of each satellite from the position of G1/G2 as a function of redshift, where coloured lines indicate the major $(>30 \%)$ and intermediate mass (between 5 and 30\%) mergers (see Table 2). The greyscales indicate minor mergers, the tone representing $f_{200}^{\text {star }}$, the stellar mass of the progenitor as a fraction of the stellar mass of the galaxy at the time of first infall into the corresponding $R_{200}$ (see Sect. 4 of the main text for full details). The shaded region indicates $R_{200}$ of G1/G2, whilst the black horizontal dashed line indicates a radius $30 \mathrm{ckpc}$, a cut which encompasses the baryonic component of the galaxy. The dotted lines follow the positions of the major progenitors after first infall within the $R_{200}$ until SUBFIND can no longer identify them as distinct objects.

is clear in both runs. In addition, G2-CS has a non-rotating, distinguishable although not-dominant bulge.

The SFRs of G1 and G2 in our two simulations, shown in the previous section (Fig. 3) and, in particular, the corresponding insitu fractions, already gave some indications of what the final morphology of the galaxy would be. In G1, star formation is extremely bursty, and the in-situ fractions are relatively low, which is indicative of a large contribution of stars from satellite galaxies. In general such a contribution from satellites will reduce the disc fraction, unless the angular momentum of the satellite is very closely aligned with that of the progenitor galaxy. In the case of G2, the smoother SFRs together with very high in-situ fractions, particularly at low redshifts, suggest the formation of a galaxy with a much more significant disc that did not suffer disruption from violent events.

In the rest of this section we show that, in fact, G1 has a much more violent merger history than $\mathrm{G} 2$ which has a much smoother evolution. It is worth noting that the in-situ fractions of our simulated galaxies are similar in runs CS and MA which is an indication that our results on the final morphologies (particularly of G1) are robust and not triggered by the assumed feedback model. Mergers seem to be as disruptive in both runs, despite differences in the exact merging times of the satellites (see below) and the different final stellar masses of the galaxies.

Figure 6 shows the merger histories of G1 and G2 in our two simulations, which we constructed by identification of haloes using the substructure finder code SUBFIND (Springel et al. 2001) and then matched between snapshots with a metric that weights intersecting particles by the ranking of their binding energy. We identified all mergers with a mass ratio larger than $0.1 \%$ in terms of their total mass, and denoted any merger greater than $30 \%$ as a major merger and any greater than $5 \%$ as an intermediate merger. Table 2 describes statistics - redshift and mass ratio - of the intermediate and major mergers as the satellites fall within the $R_{200}$ of the progenitor galaxy. We also show the mass ratios in terms of the stellar mass, as these might be more indicative of the importance of mergers in the central regions.

Whilst the accretion of a progenitor into $R_{200}$ will clearly play a role in the evolution of the galaxy, the events at much smaller radii have a more direct impact, as an infalling object can orbit the galaxy for several Gyr (or more) before merging into the central object. For this reason we followed the merging subhaloes also after their entrance into $R_{200}$, and calculated the times and mass fractions when they enter the inner $30 \mathrm{ckpc}$, where we study the stellar and gaseous components. This radius is a good choice as it is both large enough to encompass the galaxy (see Fig. 4) but also small enough that objects whose centres have passed this close will significantly interact. For both simulations, the last three columns of Table 2 show the redshift at which satellites cross the $30 \mathrm{ckpc}$ threshold, as well as the stellar mass ratio at that time. We note that, in order to match the results of runs CS and MA, we followed the mergers of satellite galaxies even though, in some cases, they have not yet merged/disrupted with the main progenitor and are still identifiable, independent systems at the present time. These are indicated in the final column of the Table 2 with the annotation "sat". We also note that 
Table 2. Properties of the major and intermediate mass mergers of G1 and G2 in our two simulations.

\begin{tabular}{|c|c|c|c|c|c|c|c|c|c|c|c|c|}
\hline & \multicolumn{6}{|c|}{ Run CS } & \multicolumn{6}{|c|}{ Run MA } \\
\hline & $z_{\mathrm{R} 200}$ & $f_{\mathrm{R} 200}^{\mathrm{tot}}$ & $f_{\mathrm{R} 200}^{\mathrm{star}}$ & $z_{30} \mathrm{ckpc}$ & $f_{30 \text { ckpc }}^{\text {star }}$ & $z=0$ & $z_{\mathrm{R} 200}$ & $f_{\mathrm{R} 200}^{\mathrm{tot}}$ & $f_{\mathrm{R} 200}^{\mathrm{star}}$ & $z_{30 \mathrm{ckpc}}$ & $f_{30 \text { ckpc }}^{\text {star }}$ & $z=0$ \\
\hline G1a & 2.52 & $67 \%$ & $51 \%$ & 2.33 & $21 \%$ & & 2.52 & $121 \%$ & $48 \%$ & 2.32 & $111 \%$ & \\
\hline G1b & 2.35 & $5 \%$ & $8 \%$ & 1.70 & $2 \%$ & & 2.35 & $6 \%$ & $8 \%$ & 2.09 & $5 \%$ & \\
\hline G1c & 1.29 & $8 \%$ & $6 \%$ & 0.82 & $5 \%$ & & 1.33 & $12 \%$ & $10 \%$ & 0.81 & $14 \%$ & \\
\hline G1d & 0.95 & $13 \%$ & $14 \%$ & 0.32 & $5 \%$ & sat & 0.95 & $13 \%$ & $33 \%$ & 0.37 & $28 \%$ & \\
\hline G1e & 0.74 & $63 \%$ & $44 \%$ & 0.28 & $27 \%$ & & 0.74 & $15 \%$ & $80 \%$ & 0.08 & $21 \%$ & sat \\
\hline G1f & 0.72 & $64 \%$ & $78 \%$ & 0.25 & $36 \%$ & & 0.70 & $19 \%$ & $108 \%$ & 0.04 & $43 \%$ & sat \\
\hline G2a & 1.48 & $11 \%$ & $11 \%$ & 0.81 & $5 \%$ & & 1.48 & $13 \%$ & $29 \%$ & 0.89 & $16 \%$ & \\
\hline G2b & 1.44 & $9 \%$ & $10 \%$ & 0.29 & $2 \%$ & sat & 1.40 & $13 \%$ & $33 \%$ & 0.44 & $9 \%$ & \\
\hline $\mathrm{G} 2 \mathrm{c}$ & 1.33 & $15 \%$ & $28 \%$ & 0.60 & $9 \%$ & & 1.33 & $18 \%$ & $61 \%$ & 0.64 & $33 \%$ & \\
\hline G2d & 1.14 & $7 \%$ & $14 \%$ & 0.03 & $5 \%$ & sat & 1.05 & $8 \%$ & $37 \%$ & 0.02 & $9 \%$ & sat \\
\hline
\end{tabular}

Notes. The G1a-f denotes the mergers for G1, and G2a-d those for G2, ordered by their appearance within the 30 ckpc. $z_{30} \mathrm{ckpc}$ and $z_{200}$ denote the highest redshift at which the most bound SUBFIND particle enters within $30 \mathrm{ckpc}$ and $R_{200}$, respectively. $f_{30 \mathrm{ckpc}}^{\text {star }}$ and $f_{\mathrm{R} 200}^{\text {star }}$ denote the SUBFIND stellar mass of the satellite as a fraction of that of the main galaxy at the corresponding redshift. $f_{\mathrm{R} 200}^{\text {tot }}$ is the total mass fraction of the satellite when it reaches $R_{200}$. For the most massive mergers (G1a, G1d, G1e, G2b) the SUBFIND mass estimate exhibits significant disruption as the object enters $R_{200}$, and so for these cases we calculate the ratios $200 \mathrm{Myr}$ earlier to avoid spurious values. In the final column, we indicate with "sat" those satellite galaxies that have not merged/disrupted at $z=0$.

the times of the mergers, particularly when the satellites reach the inner $30 \mathrm{kpc}$, are different in the two runs, as well as the merger ratios.

From Fig. 6 and Table 2 we can see that, as inferred from the growth of the haloes and the in-situ fractions, G1 has a more active history than G2, with 6 major/intermediate mass mergers, compared to only 4 in the case of G2. Perhaps more significant is that at late times $(z<1) \mathrm{G} 1$ accretes three very large objects indeed, while for G2 there are no mergers in this period (considering their entrance to $R_{200}$ ), and its only mergers for $z \in[0,3]$ are at much more modest stellar fractions. Although similar in general terms, the details of the merging process of $\mathrm{G} 1$ and $\mathrm{G} 2$ in our two runs ${ }^{8}$ show some important differences. In particular, in G1-CS only one (and small) satellite survives at $z=0$ as an independent object, while the same object has been already disrupted in run MA. More important is the fact that the two satellites that merge later (denoted G1e/f) have been disrupted by $z=0$ in run $\mathrm{CS}$ but not yet in run MA. For G2, we find less variation between the simulations and, in any case, the merger events of G2 are at much lower fractions and occur earlier compared to those of G1. For this reason, it is expected that the merger history will have a much stronger impact on the formation of G1 compared to G2.

The growth/destruction of galaxy discs is intimately related to the merger histories of galaxies. In fact, we find a clear relation between periods of disc growth/destruction and the merger history of our simulated galaxies. Figure 7 shows the evolution of $f_{\text {disc }}$, a measure of the disc-to-total (D/T) ratio, for $\mathrm{G} 1$ and $\mathrm{G} 2$ in runs CS and MA. From Fig. 5, it is clear that the distribution of circularities is a very clear indicator of the presence or absence of a disc-like stellar component and thus a single quantitative measure of this is the fraction of stellar mass with circularity larger $^{9}$ than 0.5 ,

$f_{\text {disc }} \equiv f(\epsilon>0.5)$.

8 This can explain some of the differences in the properties of G1 and $\mathrm{G} 2$ in runs CS and MA, e.g. morphologies, that we discussed in this and the previous sections.

9 In the calculation of $f_{\text {disc }}$, we consider only particles of the main subhalo, excluding satellites.
We note, however, that $f_{\text {disc }}$ can be non-zero even if there is no disc, if the distribution of circularities is sufficiently broad. On the other hand, previous work (Scannapieco et al. 2010; see also Abadi et al. 2003; Governato et al. 2007) showed that kinematic $\mathrm{D} / \mathrm{T}$ decompositions, as the one we present here, give ratios that are systematically and significantly lower than those obtained with photometric decompositions (i.e. comparable with observed D/T ratios). For example, in Scannapieco et al. (2010) we showed that $\mathrm{D} / \mathrm{T}$ can increase from 0.2 (in a kinematic decomposition) up to $0.5-0.7$ (in a photometric decomposition). In any case, as the primary focus of our analysis is to find an appropriate measure of the evolution of a galaxy's morphology (and not to make a detailed comparison of simulated and observed $\mathrm{D} / \mathrm{T}$ ratios), we can safely use $f_{\text {disc }}$ as it sufficiently captures the prominence of the disc as a function of time in our galaxies.

The more violent merger history of G1 is also clear from the evolution of $f_{\text {disc }}$, as G1 exhibits significant morphological evolution between $z=3$ and $z=0$. Most of the rotational support of $\mathrm{G} 1$ is acquired between $z \sim 2$ and $z \sim 1.5$ in both runs. In G1CS, the disc is stable until $z \sim 0.3$, when it is disrupted coinciding with the merger events occurring at $z \sim 0.3-0.1$. For G1-MA, we find an important decrease in $f_{\text {disc }}$ between $z=1.5$ and $z \sim 0.5$, and a second phase of disc growth after $z \sim 0.4$. Despite the differences in the evolution of G1-CS and G1-MA, mainly driven by the different merger times of the satellite galaxies (when they enter the inner regions), in both simulations we find a galaxy where the rotational support, when present, cannot be maintained for long periods of time.

The more peaceful merger history of the G2 halo is consistent with the rearing of a more disc-dominated galaxy, and a more continuous and smooth disc growth. In both simulations, G2 evolves into a galaxy with a significant disc, little affected by disruptive merger events. An important difference is however the typical age and formation time of the discs in the two simulations: the disc of G2-CS is present from very early times, and therefore is older on average, while in G2-MA the disc does not form until after $z=1$ and never reachs $f_{\text {disc }}$ values as large as 

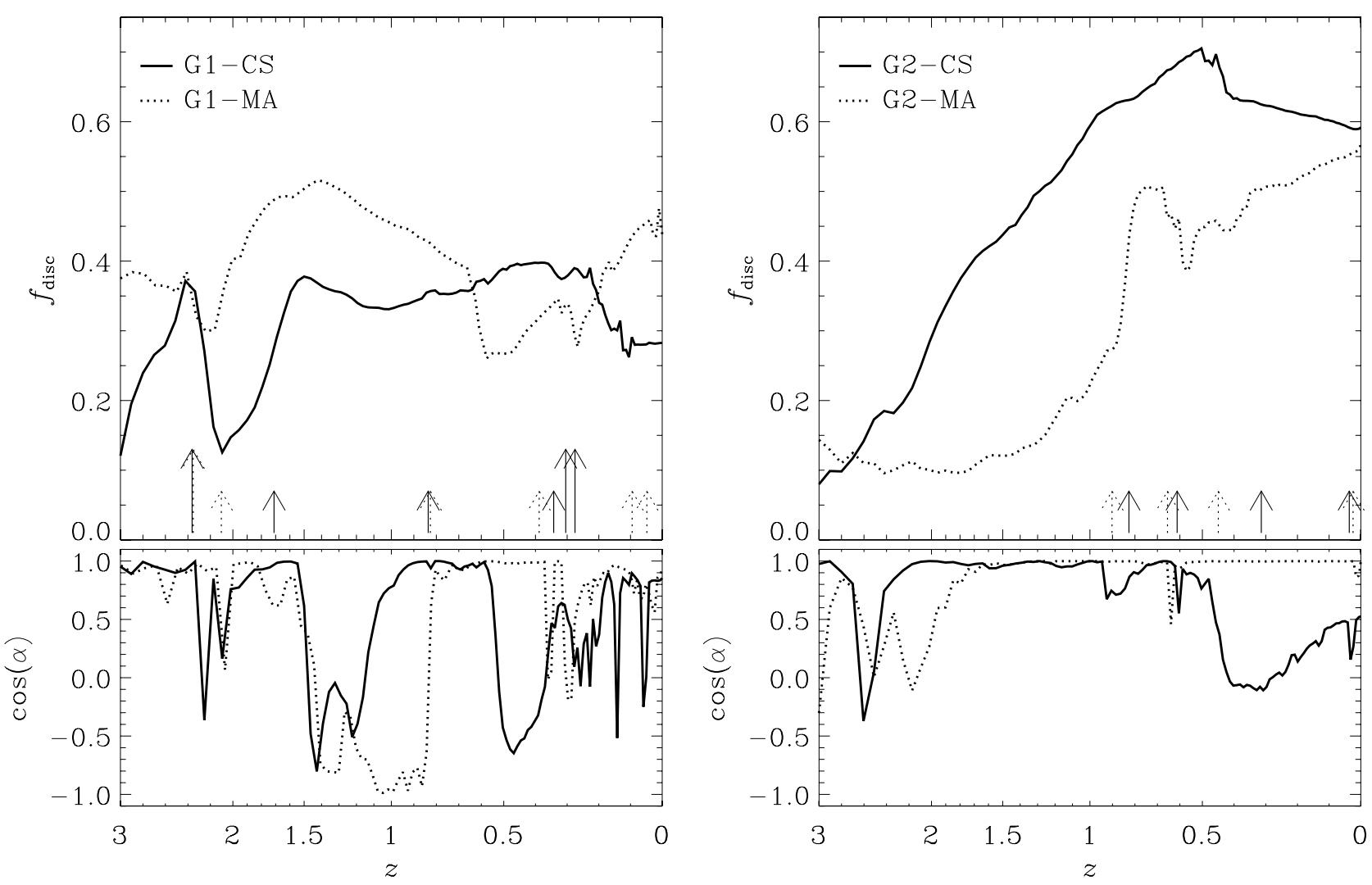

Fig. 7. Evolution of the disc-to-total measure $f_{\text {disc }}$ of G1 and G2 in our two simulations. The long/short arrows denote the entrance of major/intermediate mass mergers in the inner $30 \mathrm{ckpc}$ in the two runs. The lower panels show the evolution of $\cos (\alpha)$, the angle between the corresponding angular momentum vectors of the gas and stars.

those found for G2-CS. This is, as explained before, the result of the stronger feedback assumed in the MA model, which produces a lower star formation activity at early times owing to the prompt effects of radiation pressure and kinetic feedback from young stars.

Finally, in the lower panels of Fig. 7 we show the corresponding evolution of the cosine of the angle $\alpha$ between the specific angular momenta of the gas $\left(\boldsymbol{j}_{\text {gas }}\right)$ and of the stars $\left(\boldsymbol{j}_{\text {stars }}\right)$, namely

$\cos (\alpha) \equiv \hat{\boldsymbol{j}}_{\text {stars }} \cdot \hat{\boldsymbol{j}}_{\text {gas }}$,

where the hat denotes a normalized vector. In previous work, we found that if the angular momentum vectors of the gas and the stars are misaligned, the stellar discs become unstable and the D/T ratios inevitably decrease (Scannapieco et al. 2009). Such a misalignment can occur as a consequence of merger events, but also if accreting gas brings angular momentum in a different direction than that of the pre-existing stellar disc.

Consistent with our previous analysis, we find that the alignment between the gas and stars is not well preserved during the evolution of G1, mainly as a consequence of the major merger events of this galaxy, which determine the end points of the growth of the discs. The results are very similar for our two runs, which is again an indication that results are robust and not affected by the particular feedback assumed but rather by the merger history. In the case of G2, the gas and the stars are much better aligned at all times, in both runs. The most important difference in this case is at late times, when G2-CS shows a strong misalignment, consequence of the mergers that are taking place at $z \lesssim 0.5$. As seen before, in G2-MA one of such satellites has already been disrupted while the other has not yet reached the central object.

In summary, by comparing these two histories we see a picture where the discs are built-up during periods of absence of major mergers and alignment between the gaseous and stellar discs. In both simulated galaxies the growth of the discs suffers termination events when such alignment is disrupted, usually associated with merging satellites (but can also be produced by misaligned gas accretion), either when they pass within $30 \mathrm{ckpc}$ or merely have a close approach.

\section{Discussion and conclusions}

We used a simulation of the Local Universe where a Local Group-like pair of galaxies with large-scale environment constrained to reproduce the environment of the actual LG forms. We study how the two most massive galaxies within the simulated LG, candidates for the Milky Way and Andromeda, formed and evolved. The constrained nature of the simulation is relevant (Creasey et al. 2015), as it allows the study of the evolution of galaxies in an environment similar to our LG, and thus the investigation of how it affects the properties of the galaxies along cosmic time.

We run two simulations using the same initial conditions, but assuming different feedback prescriptions, in order to assess whether our results might in any sense be affected by the particular choice of feedback model. It is well known that the detailed properties of simulated galaxies depends on the implementation of the physical processes included, in particular on 
the way stellar feedback is treated (Scannapieco et al. 2012). Furthermore, it is still not possible to select the several input parameters of the simulation from physical principles, and various numerical/technical choices can also affect the final outcome of simulations. Despite these problems, which will certainly witness significant progress in the next few years, the predictions of simulations in relation to the evolution of properties such as morphologies should be still reliable.

In our simulations, the two main galaxies of the simulated LG, G1 (candidate for M31) and G2 (candidate for MW), exhibit rather different formation and merger histories although they are, at $z=0$, both members of the same LG. G1 is much more active in terms of the frequency and mass ratios of its mergers compared to G2. More importantly, G1 experiences three major/intermediate mass mergers at $z<1$, contrary to G2 that has (since $z=3$ ) only four intermediate mass mergers, and these occur before $z=1$. The merger rates of the two galaxies are then a first indication that the two galaxies will follow different evolutionary paths, in particular in relation to the formation/destruction of stellar discs.

In fact, we find that $\mathrm{G} 1$, having 6 intermediate/major mergers between $z=3$ and $z=0$, cannot grow a stellar disc that survives until the present time. The various mergers disturb G1 significantly, changing the amount and orientation of the angular momentum of the stars and gas, which in both codes leads to a posterior misalignment of the two vectors. When such a misalignment is present, any pre-existing disc gets fully or partially destroyed. In contrast, the much more smooth merger history of G2, where the angular momentum of the stars and gas are very well aligned during most of the time, is consistent with the formation of an extended, rotationally supported disc-like galaxy. It is worth noting that mergers, even fairly sizeable ones, do not necessarily destroy discs, and the actual damage depends on the orbital parameters (see also Wang et al. 2012).

The different merger/formation histories of G1 and G2 is also reflected in their star formation rates and in-situ fractions. G1 has a much more violent SFR, and low in-situ fractions, indicating a large contribution of stellar material from infalling satellites. With such an active merger history, it seems very hard to grow a stellar disc with the satellites feeding the disc, instead of destroying it. The more peaceful history of G2 and its much higher in-situ fractions at all times suggest that this galaxy will have a much higher chance of disc survival.

We investigated and compared the evolution of the MW and M31 candidates in two runs, a first one where only supernova feedback is considered (CS) and a second one that additionally includes the effects of radiation pressure from massive stars and a kinetic component for the supernova energy (MA). This comparison allowed us to assess the robustness of our results and their dependence on the assumed feedback model. While it is true that, owing to the larger feedback effects, the MA model produces galaxies with reduced stellar masses compared to CS, both for the progenitor and for the satellites, mergers are as disruptive in the MA model as in the CS simulation, where galaxies have in general larger stellar masses. In both simulations we find that the stellar disc of G1 suffers several episodes of partial destruction that follow merger events, while in G2 a stellar disc grows steadily as a result of its more quiescent evolution. We found (expected) differences in the exact merger times of satellites between the two runs, not only when they enter $R_{200}$ but more importantly when they reach the inner galaxy. These can cause some of the satellites to be, at any given time, already disrupted in one run but not in the other. More important are the overall differences in the star formation rates in terms of the assembly of the stellar components: the galaxies in CS are systematically older than in MA, which is a direct result of the stronger feedback assumed in the latter.

In summary, by comparing the histories of formation of G1 and $\mathrm{G} 2$, and its relation to the formation/destruction of stellar discs along cosmic time, we conclude that the rearing of a disc can only be produced during quiet periods where no significant mergers and/or close encounters are produced, and when the angular momentum vectors of the stars and the gas are well aligned. These results are consistent with those found earlier in S09, where we simulated the formation of eight galaxies in a more isolated environment, giving support to the idea that it is the particular formation history of haloes at relatively small scales (smaller at least than the LG) that determines their morphological changes along cosmic time, rather than their membership of a loose group such as the LG.

Acknowledgements. We thank the reviewer for his/her constructive suggestions. We thank M. Aumer for fruitful discussions on the implementation of radiation pressure and kinetic feedback on the simulation code. The simulation was performed on the Juropa supercomputer of the Jülich Supercomputing Centre (JSC), and initial tests were run at the Barcelona Supercomputer Centre (BSC). C.S. and P.C. acknowledge support from the Leibniz Gemeinschaft through grant SAW-2012-AIP-5 129. C.S. acknowledges support from the HPC-EUROPA2 project, with the support of the European Community Research Infrastructure Action of the FP7. S.E.N. acknowledges support from the Deutsche Forschungsgemeinschaft under the grants MU 1020 16-1 and NU 332/2-1, and G.Y. thanks MINECO (Spain) for supporting his research through different projects: AYA2012-31101, FPA2012-34694 and Consolider Ingenio SyeC CSD2007-0050.

\section{References}

Abadi, M. G., Navarro, J. F., Steinmetz, M., \& Eke, V. R. 2003, ApJ, 591, 499

Agertz, O., \& Kravtsov, A. V. 2014, ApJ, submitted [arXiv: 1404 . 2613]

Agertz, O., Kravtsov, A. V., Leitner, S. N., \& Gnedin, N. Y. 2013, ApJ, 770, 25

Aumer, M., White, S. D. M., Naab, T., \& Scannapieco, C. 2013, MNRAS, 434, 3142

Bahé, Y. M., McCarthy, I. G., Balogh, M. L., \& Font, A. S. 2013, MNRAS, 430, 3017

Bird, J. C., Kazantzidis, S., Weinberg, D. H., et al. 2013, ApJ, 773, 43

Blanton, M. R., Eisenstein, D. J., Hogg, D. W., et al. 2003, BAAS, 36, 589

Bovy, J., Allende Prieto, C., Beers, T. C., et al. 2012, ApJ, 759, 131

Ceverino, D., Klypin, A., Klimek, E. S., et al. 2014, MNRAS, 442, 1545

Christensen, C., Quinn, T., Governato, F., et al. 2012, MNRAS, 425, 3058

Corbelli, E., Lorenzoni, S., Walterbos, R., Braun, R., \& Thilker, D. 2010, A\&A, 511, A89

Creasey, P., Theuns, T., \& Bower, R. G. 2013, MNRAS, 429, 1922

Creasey, P., Scannapieco, C., Nuza, S. E., et al. 2015, ApJ, 800, L4

Dressler, A. 1980, ApJ, 236, 351

Fall, S. M., \& Efstathiou, G. 1980, MNRAS, 193, 189

Few, C. G., Gibson, B. K., Courty, S., et al. 2012, A\&A, 547, A63

Girardi, M., Rigoni, E., Mardirossian, F., \& Mezzetti, M. 2003, A\&A, 406, 403

Gottlöber, S., Hoffman, Y., \& Yepes, G. 2010, Proc. High Performance Computing in Science and Engineering, Garching/Munich 2009 (Springer) [arXiv: 1005.2687]

Governato, F., Willman, B., Mayer, L., et al. 2007, MNRAS, 374, 1479

Guzzo, L., Strauss, M. A., Fisher, K. B., Giovanelli, R., \& Haynes, M. P. 1997, ApJ, 489, 37

Haardt, F., \& Madau, P. 1996, ApJ, 461, 20

Hermit, S., Santiago, B. X., Lahav, O., et al. 1996, MNRAS, 283, 709

Hopkins, P. F., Kereš, D., Oñorbe, J., et al. 2014, MNRAS, 445, 581

Kafle, P. R., Sharma, S., Lewis, G. F., \& Bland-Hawthorn, J. 2012, ApJ, 761, 98

Kim, J.-h., Abel, T., Agertz, O., et al. 2014, ApJS, 210, 14

Murante, G., Monaco, P., Borgani, S., et al. 2015, MNRAS, 447, 178

Navarro, J. F., \& Benz, W. 1991, ApJ, 380, 320

Nuza, S. E., Dolag, K., \& Saro, A. 2010, MNRAS, 407, 1376 
Nuza, S. E., Kitaura, F.-S., Heß, S., Libeskind, N. I., \& Müller, V. 2014a, MNRAS, 445, 988

Nuza, S. E., Parisi, F., Scannapieco, C., et al. 2014b, MNRAS, 441, 2593

Okamoto, T., Eke, V. R., Frenk, C. S., \& Jenkins, A. 2005, MNRAS, 363, 1299

Piffl, T., Scannapieco, C., Binney, J., et al. 2014, A\&A, 562, A91

Piontek, F., \& Steinmetz, M. 2011, MNRAS, 410, 2625

Quinn, P. J., Hernquist, L., \& Fullagar, D. P. 1993, ApJ, 403, 74

Sales, L. V., Navarro, J. F., Theuns, T., et al. 2012, MNRAS, 423, 1544

Scannapieco, C., Tissera, P. B., White, S. D. M., \& Springel, V. 2005, MNRAS, 364,552

Scannapieco, C., Tissera, P. B., White, S. D. M., \& Springel, V. 2006, MNRAS, 371, 1125

Scannapieco, C., Tissera, P. B., White, S. D. M., \& Springel, V. 2008, MNRAS, 389,1137

Scannapieco, C., White, S. D. M., Springel, V., \& Tissera, P. B. 2009, MNRAS, 396, 696 (S09)

Scannapieco, C., Gadotti, D. A., Jonsson, P., \& White, S. D. M. 2010, MNRAS, 407, L41
Scannapieco, C., White, S. D. M., Springel, V., \& Tissera, P. B. 2011, MNRAS, 417,154

Scannapieco, C., Wadepuhl, M., Parry, O. H., et al. 2012, MNRAS, 423, 1726

Sellwood, J. A., \& Binney, J. J. 2002, MNRAS, 336, 785

Smith, M. C., Ruchti, G. R., Helmi, A., et al. 2007, MNRAS, 379, 755

Springel, V. 2005, MNRAS, 364, 1105

Springel, V., White, S. D. M., Tormen, G., \& Kauffmann, G. 2001, MNRAS, 328,726

Springel, V., Wang, J., Vogelsberger, M., et al. 2008, MNRAS, 391, 1685

Steinmetz, M., \& Navarro, J. F. 1999, ApJ, 513, 555

Stinson, G. S., Bovy, J., Rix, H.-W., et al. 2013, MNRAS, 436, 625

Trujillo-Gomez, S., Klypin, A., Colín, P., et al. 2015, MNRAS, 446, 1140

Vogelsberger, M., Genel, S., Sijacki, D., et al. 2013, MNRAS, 436, 3031

Wang, J., Hammer, F., Athanassoula, E., et al. 2012, A\&A, 538, A121

Watkins, L. L., Evans, N. W., \& An, J. H. 2010, MNRAS, 406, 264

Wilkinson, M. I., \& Evans, N. W. 1999, MNRAS, 310, 645

Yepes, G., Gottlöber, S., \& Hoffman, Y. 2014, New Astron. Rev., 58, 1

Ziparo, F., Popesso, P., Biviano, A., et al. 2013, MNRAS, 434, 3089 